\title{
Neutron spectroscopy: an under-utilised tool for organic electronics research?
}

\author{
Hamish Cavaye
}

\section{Published version information}

This is the peer reviewed version of the following article:

Citation: H Cavaye. "Neutron spectroscopy: an under-utilised tool for organic electronics research?" Angewandte Chemie International Edition, vol. 58, no. 28 (2019): 9338-9346.

DOI: $10.1002 /$ anie.201812950

Which has been published in final form at DOI above. This article may be used for non-commercial purposes in accordance with Wiley-VCH terms and conditions for self-archiving.

Please cite only the published version using the reference above. This is the citation assigned by the publisher at the time of issuing the AAM. Please check the publisher's website for any updates. 


\title{
Neutron Spectroscopy: An Under-Utilised Tool for Organic Electronics Research?
}

\author{
Hamish Cavaye ${ }^{1, *}$ \\ ${ }^{1}$ ISIS Neutron and Muon Source, Rutherford Appleton Laboratory, Science and Technology Facilities Council, \\ Didcot, $O X 110 Q X, U K$ \\ *Corresponding author: hamish.cavaye@stfc.ac.uk
}

\begin{abstract}
Neutron scattering is a well established technique that has proven to be an invaluable tool in myriad fields of chemical and physical research. Neutrons offer unique ways to study in-situ or operando functional materials due to their highly penetrating nature and specific interactions with the nuclei of different isotopes. While some neutron scattering techniques, such as neutron diffraction (ND), neutron reflectometry (NR), and small-angle neutron scattering (SANS), have already been heavily adopted by the scientific community for use in the research of organic electronics, there are a number of techniques that are far less widely used; spectroscopic neutron scattering. This article aims to highlight these "under-utilised" techniques, to emphasise their potential use within the field of organic electronics, and to increase awareness of their utility among new research communities.
\end{abstract}

\section{Introduction}

Neutrons are a powerful probe for the chemical and physical properties of materials. [1,2] In a neutron scattering experiment, the intensity of scattered neutrons is measured as a function of scattering angle after interaction with the sample of interest. This interaction can be either inelastic or elastic; with or without the exchange of energy between the neutron and the sample respectively. In very broad terms, elastic scattering of neutrons provides information regarding the structure of a sample whereas inelastic scattering provides information regarding the motions of atoms or groups of atoms within a sample.

Both X-ray diffraction and neutron diffraction (ND) are ubiquitous and their discussion is out of the scope of this article. Neutron reflectometry (NR) and small-angle neutron scattering (SANS) are two neutron scattering techniques that have already become quite well-adopted by the organic electronics research community. Many organic electronic devices comprise a stacked structure with numerous layers that have thicknesses appropriate to study with NR. Other common features of organic electronics are blends of two or more com- ponents, self-assembly, and nano-scale structures of separated phases. SANS offers a convenient probe into the size, shape, and composition of such structures.

All of the neutron scattering methods mentioned above are used exclusively to obtain structural information about the sample in question. In contrast, and complimentary to these structural methods, molecular spectroscopy with neutrons is well-established in the broader literature as a method to obtain information regarding the dynamics of a system. 3, 4 The most common neutron spectroscopy techniques are inelastic neutron scattering (INS) and quasielastic neutron scattering (QENS). INS is used to study vibrational spectra and QENS is used to examine molecular motion and diffusion. Structural techniques such as ND, NR, and SANS are well established in the field of organic electronics whereas spectroscopic measurements are significantly less well represented.

\subsection{Neutron spectroscopy}

Neutron spectroscopy has a number of key features or properties that make it unique among other spectroscopic methods. Key property 1 is that unlike photons, which primarily interact with 
electrons, neutrons interact with the nucleus of an atom. Different elements and isotopes can have dramatically different neutron scattering powers and the intensity of the spectroscopic response is directly proportional to the scattering power of the atoms involved. [5] This means that light atoms can be examined in the presence of heavy ones and isotopic labelling can offer a handle to improve contrast.

Following on from this, key property 2 is that the well documented scattering powers of atoms means that neutron scattering spectra are relatively simple to simulate and predict. This means the techniques are ideal either as methods of validating theoretical models or, conversely, theoretical modelling can be utilised in order to more fully elucidate the measured data. It is important to note, however, that simulations involving highly disordered or amorphous materials can require approximations, such as isolated molecule calculations, to be used.

Key property 3 is that neutrons are very highly penetrating. That is, neutron spectroscopy is a "bulk sample" technique and is ideal for studying bulk properties of materials as well as materials that are encapsulated within bulky sample environments or within operando device architectures. The weak interaction between neutrons and most elements also means that it is rare to damage the sample by exposure to neutrons, which can be a problem with other probes such as lasers or X-rays.

\subsection{Key questions in organic electronics research}

The field of organic electronics research comprises a number of key questions or interest areas in which neutron spectroscopy can be a vital tool. In no particular order these include but are not limited to:

- Low-energy intermolecular vibrational modes, which are important for understanding the dynamics of a system relating to electron transport.

- How the dynamics of a sample contribute to the structure-property relationships in conducting organics.

- Understanding of (unwanted) excited-state relaxation mechanisms.
- Sample or solution dynamics during device fabrication and how they relate to device performance.

- Degradation mechanisms in devices, eg. those caused by dopant diffusion.

This mini-review aims to briefly introduce the spectroscopic neutron scattering techniques INS and QENS to readers that are currently unaware of them, and to highlight the successful use of these methods within the wider field of organic electronics to shed light on the key questions posed herein. It is hoped that this article will increase the awareness of such techniques, and broaden their use.

\section{Inelastic Neutron Scattering (INS)}

INS is a technique used to study the vibrational spectra of molecular and crystalline components of a system. During an INS experiment a neutron of kinetic energy $E_{i}$ collides with a sample, transferring some of its energy to excite vibrational modes within the sample, and is scattered with kinetic energy $E_{f}$. The difference $E_{i}-E_{f}$ corresponds to the energy of the excited vibration and thus a spectrum can be measured. For a more in-depth discussion of the phenomena behind generating an INS spectrum the book by Mitchell, et al. is comprehensive and very readable. [6]

These neutron vibrational spectra are directly analogous to those measured using photons, such as IR and Raman spectroscopy, however the transition energies remain unchanged and only the peak intensities differ. This means that INS is highly complementary to these photon-based techniques. INS is sensitive to all vibrational modes because optical selection rules do not apply. This means that a single INS spectrum can provide information about modes that would be inactive in either an IR or Raman spectrum, or even those modes that are inactive in both. Additionally, the intensity of modes in an INS spectrum is directly related to the neutron scattering power of the nuclei involved. This not only means that compared to IR and Raman the spectra from INS can be relatively easy to calculate and predict, but also that selective isotopic labelling can control both the intensity and 

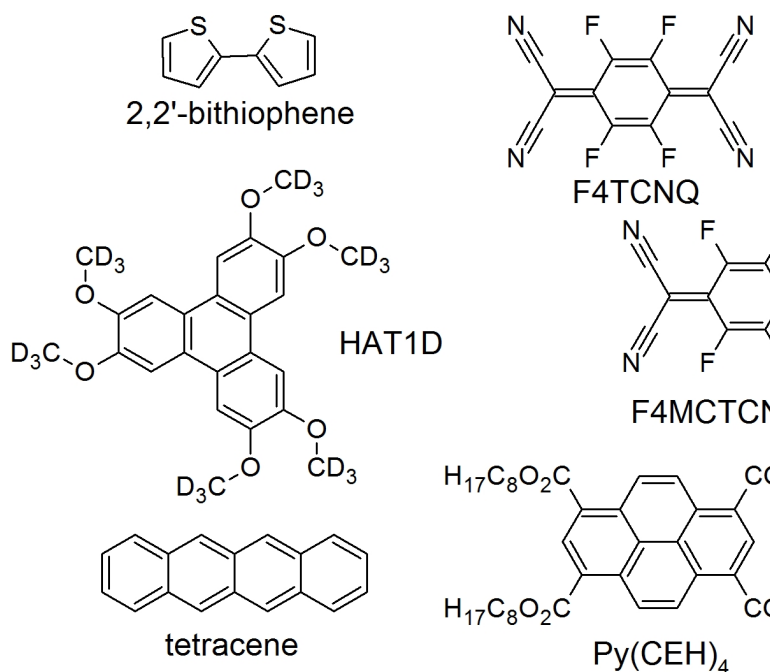

F4TCNQ
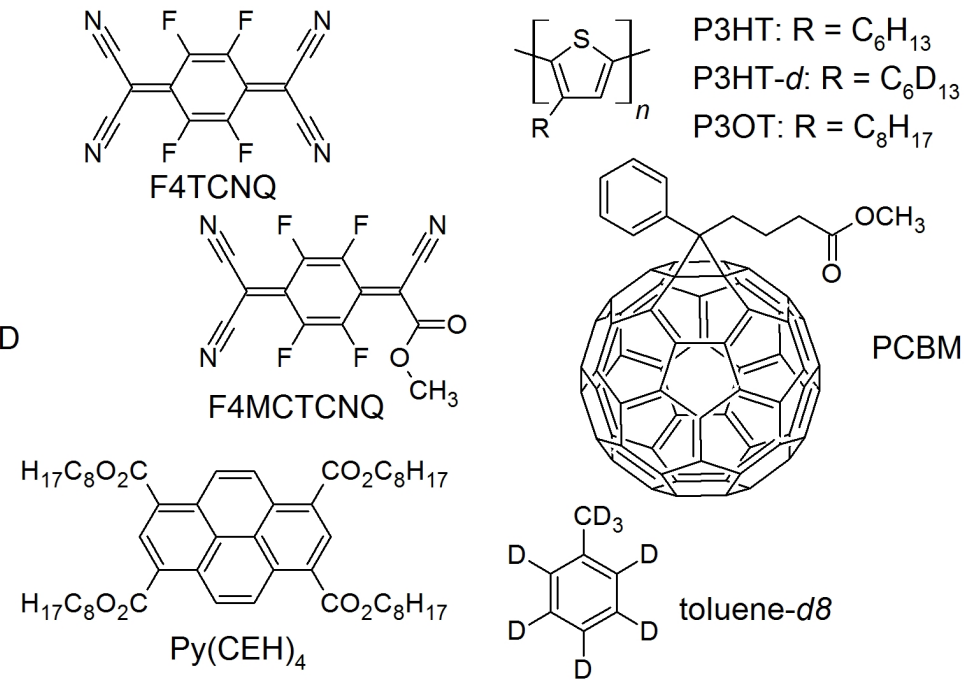

Figure 1: Chemical structures for some of the materials discussed in this article.

the frequency of chosen modes within the sample. Finally, unlike IR and Raman which are most commonly used to examine the surface of a sample, the penetrating nature of the neutrons means INS is ideal for studying bulk properties of materials.

\subsection{INS and organic electronics}

The ability to accurately predict INS spectra means that INS can be used to validate theoretical analyses of conducting organic systems. Hermet, et al. studied the phonon density of states in crystalline 2,2'-bithiophene (Figure 1), a reference compound for conjugated polymers, using $a b$ initio calculations. 7 The investigation of these low-energy intermolecular modes is important for fully understanding the dynamics of the system relating to electron transport, one of the key areas of interest mentioned above. The study compared experimental INS spectra with simulations and showed that low-frequency $\left(40-320 \mathrm{~cm}^{-1}\right)$ phonon modes could only be accurately simulated by increasing the size of the crystalline unit cell. The simulated spectrum only afforded very good agreement with the experimental INS spectrum when using a $2 \times 2 \times 2$ "supercell" of 8 unit cells. This provided the group with a lower-bound for the size of a calculation necessary to afford useful predictions.

In 2009 the same group used a combination of far-infrared absorption and INS to study four new oligo(phenylenethienylene)s, potentially useful materials as semiconductors for organic field-effect transistors. 8 By comparing the far IR spectra, the INS spectra, and first-principles calculations of each of the structurally related oligomers, the researchers were able to reliably assign the inter- and intramolecular vibrational modes. These results help to build structure-property relationships for new conducting organic materials, which is vital for future improvements in performance.

Electron-phonon coupling in a self-assembling discotic material was the topic of a study by Kruglova, et al. 9 The electron-phonon coupling underlies unwanted excited state relaxation in these organic molecules, which function not only as hole conductors, but also as light-absorbing dyes. Understanding this unwanted decay of excited states is another of the key areas of interest within organic electronics research. The group studied a family of related triphenylene derivatives, each comprising alkoxy substitents of different lengths, again with the aim of understanding the minimum theoretical model necessary to accurately describe the vibrational dynamics of the system. Selective deuteration of the alkoxy tails allowed the vibrational modes of the cores of the molecules to be studied in isolation, despite the varying tail lengths. This is because the incoherent neutron scattering power of ${ }^{2} \mathrm{H}$ atoms is many times lower than that of a ${ }^{1} \mathrm{H}$ atom, meaning the intensity of modes involving deuterated moieties can be significantly reduced. Figure 1 shows the chemical structure of one such partially discotic material, HAT1D, 


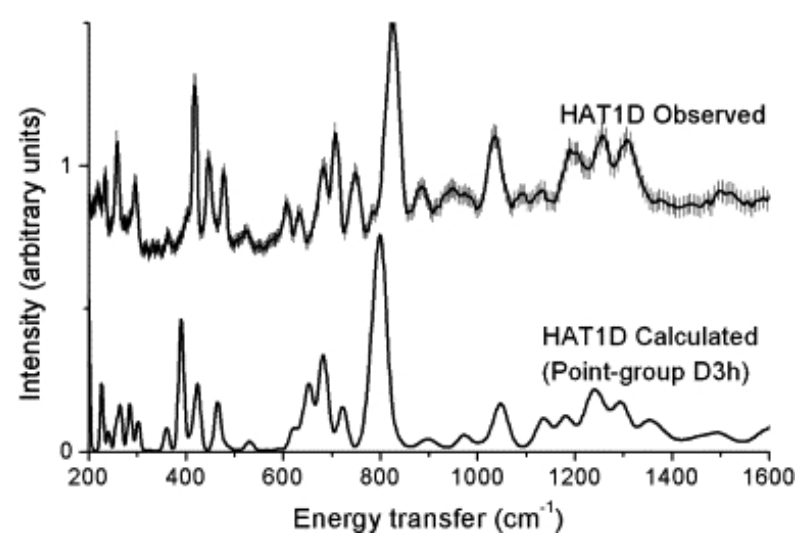

Figure 2: The observed (top) and calculated (bottom) INS spectra for HAT1D, emphasising the ability to readily simulate neutron spectra theoretically. Reproduced with permission from ref. 9 .

and the observed and simulated INS spectra for HAT1D can be seen in Figure 2, It was found that the alkoxy tails had only a slight effect on the vibrational dynamics of the triphenylene cores and replacement of the alkoxy group by $\mathrm{OH}$ within the simulations allowed the vibrational characteristics of interest to be reproduced sufficiently for further theoretical study at reduced computational cost.

The highly penetrating nature of neutrons was exploited by Pivovar, et al. in a study of inter- and intramolecular interactions in crystalline tetracene (Figure 1) as a function of applied hydrostatic pressure. [10] INS spectra were recorded at pressures of up to $358 \mathrm{MPa}$; assignment of the peaks was aided by a combination of $a b$ initio calculations and molecular dynamics simulations. It was found that some peaks shifted to higher energy with increasing pressure, whereas other peaks were largely unchanged (see Figure 3). Further analysis indicated that the application of pressure had an anisotropic effect on the vibrational modes of tetracene. Changes in energy were mainly observed for intramolecular vibrational modes with atomic displacements perpendicular to the molecular plane and intermolecular modes with displacements in the $a b$-plane of the herringbone layers of the crystal. Better understanding of these molecular interactions is vital to improving charge-transport properties of devices.

In 2017, Harrelson, et al. used INS to study atomic scale structure in semicrystalline poly(3hexylthiophene) (P3HT, Figure 1) both with and without the dopant molecule 2,3,5,6-tetrafluoro-

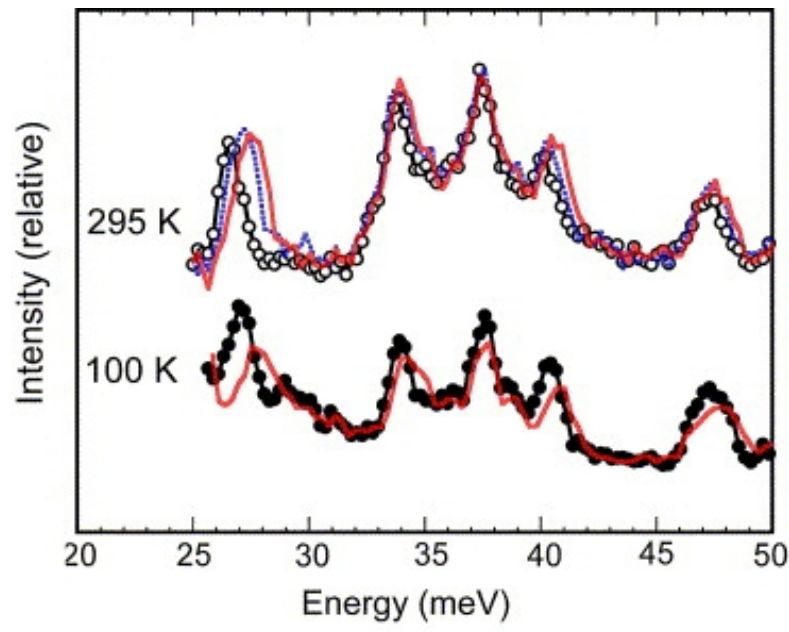

Figure 3: Ambient and high pressure experimental INS spectra of tetracene collected at $295 \mathrm{~K}$ (top) and at $100 \mathrm{~K}$ (bottom). The ambient pressure data are depicted by open circles (o) at $295 \mathrm{~K}$ and filled circles $(\bullet)$ and $100 \mathrm{~K}$. The spectra collected at 218 and $358 \mathrm{MPa}$ are indicated by the dashed black line (295 K only) and solid red line, respectively. It is clear to see that some modes are affected by the changes in pressure whereas others are not. Reproduced with permission from ref. [10].

7,7,8,8-tetracyanoquinodimethane

(F4TCNQ, Figure 11). [11] The group took advantage of the lack of selection rules and quantitatively decomposed the INS spectra, elucidating the dominant structural motifs in both the doped and undoped samples despite significant structural heterogeneity. By comparing their experimental results with $a b$ initio calculations, Harrelson and colleagues were able to show that F4TCNQ was predominantly intercalated between the $\mathrm{P} 3 \mathrm{HT}$ chains, causing the crystalline regions of the polymer chain to become flatter. This structural change is thought to be a significant factor impacting the electrical properties of the doped semi-conductor. It is worth noting that despite neutron spectroscopy being a probe of molecular dynamics, in this example it provided structural information as well. The group also note that while the INS spectra were of sufficiently high resolution to infer detailed structural characteristics of disordered systems, the ability to model semi-crystalline and doped semi-conductor polymers is limited by modern methodology and computational power. This in turn limits the ability to accurately model lower energy/longer range vibrational modes and needs to be an area of improvement in the future. 
As recently as 2018, the same group again utilised INS to directly probe the nuclear modes that might be limiting charge mobility in molecular semiconductors. 12 In this study the focus was on the abilty of INS to resolve the low-energy phonon spectrum in crystalline samples of benzothienobenzothiophene derivatives. These phonon spectra were then used to validate theoretical predictions of electron-phonon coupling parameters, leading to high quality predictions of hole mobilities for the materials being studied, which is a primary figure of merit in p-type semiconductors. Importantly the researchers summarise that while their work involved charge transport in organic transistor materials, the vibrational dynamics being studied are of import to other branches of organic electronics such as exciton separation in organic photovoltaics and dynamical processes in organic LEDs.

The work discussed in this section illustrates the potential for INS in organic electronics research, however it is limited in quantity. There are myriad conducting organic materials that would benefit from investigation with INS to address the key questions posed above.

\section{Quasielastic Neutron Scatter- ing (QENS)}

QENS is a technique used to study the dynamics of atoms or ions (or groups thereof) within a sample. Much like in an INS experiment, neutrons are scattered from the sample and measured to afford a spectrum of neutron energy change, $\Delta E$, after interaction with the sample. Of primary interest for QENS, however, is the region of this spectrum immediately surrounding the elastic peak, that is, the peak of zero energy change after scattering. An example of such an elastic peak can be seen in Figure 5, centred on $\Delta E=0$. Motion of the atoms or ions in the sample when they scatter an incident neutron cause a Doppler broadening of this elastic peak, and thus a lessening of its intensity and an increase in the intensity of the background signal. Additionally, when the angle of neutron scattering is taken into account at the same time as energy transfer then the full momentum transfer dependence or " $Q$ "-dependence of the spectrum can be analysed. The changes in the shape and intensity of the elastic peak versus temperature and
$Q$ can be compared with well-established models of atomic motion to afford not only rates of atomic motion but also distances travelled and activation energies, both of which are difficult to achieve with other techniques. A more comprehensive and very well-written introduction to QENS that is suitable for newcomers was published by Embs, et al. in 2010 [4.

QENS naturally lends itself to examining the dynamics of organic materials. This is due to the large incoherent neutron scattering power of ${ }^{1} \mathrm{H}$ atoms, which are ubiquitous in organic materials, affording a strong signal for their motions. Another important aspect of QENS is the energy resolution and "dynamic window" offered by the spectrometer being used. Dynamic processes in the sample that are too fast or too slow will fall outside of the QENS window and will not be observed. The majority of QENS spectrometers cover timescales in the range of $10^{-13}-10^{-9} \mathrm{~s}$ and length scales on the order of 1-30 A, 13 thus making them suitable for studying phenomena such as polymer relaxation and ionic diffusion, both of which can be important in organic electronics. Another strength of QENS is its use to validate molecular dynamics (MD) models. Such MD simulations are extremely useful to investigate properties of the materials used in organic electronics, however their accuracy is often questioned. Use of QENS data can provide a stringent test of the model.

\subsection{QENS and organic electronics}

In 2009 Obrzut and Page were the first to examine a functional photovoltaic material using QENS when they looked at relaxation processes in conducting regioregular P3HT. [14] The researchers took advantage of the high incoherent scattering cross-section of hydrogen atoms to selectively monitor the contribution of the side chains to molecular dynamics in the polymer. Figure 4 a shows a "fixed elastic window scan", which plots the intensity of elastically scattered neutrons against temperature. Changes in gradient can indicate motions becoming active or inactive. By comparing the mean square displacement (Figure $4 \mathrm{~b}$ of the protons as a function of temperature it was shown that the hexyl side chains are liberated at approximately $175 \mathrm{~K}$, consistent with findings that long-range order in electronic states would 


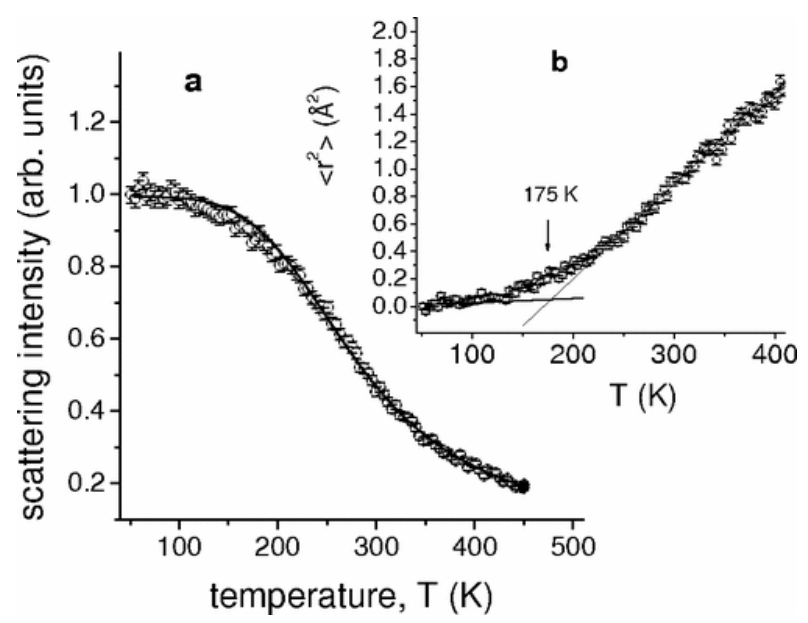

Figure 4: (a) Normalized elastic-scattering intensity as a function of temperature (at fixed $\mathrm{Q} \approx 0.99 \AA^{-1}$ ). The solid line represents a fit of the data using an instrument resolution of $0.86 \mathrm{meV}$ as determined from the dynamic scan at $50 \mathrm{~K}$. (b) Mean-square displacement, $\left\langle r^{2}\right\rangle$, for P3HT as measured by QENS showing the onset of motions above $175 \mathrm{~K}$. Reproduced with permission from ref. 14 .

approach that of amorphous silicon below $180 \mathrm{~K}$. This relaxation of side groups was shown to contribute to topological disorder, hindering charge transport, and decreasing conductivity by 5 orders of magnitude at $190 \mathrm{~K}$.

In 2013, Paternó, et al. built upon the work by Obrzut and Page by using QENS to study solid blends of $50 \mathrm{wt} \%$ P3HT and [6,6]-phenyl$\mathrm{C}_{61}$-butyric acid methyl ester (PCBM, Figure 1) cast from different solvents with a range of boiling points. 15] This ratio of P3HT:PCBM is directly relevant to bulk heterojunction photovoltaic cells. The group found that the solvent with the intermediate boiling point, chlorobenzene, afforded the most long range order to the blends, with the greatest level of crystallinity observed. However, despite this fact, when the dynamics of the blends were investigated at around $285 \mathrm{~K}$, the glass transition temperature of $\mathrm{P} 3 \mathrm{HT},[16$ no difference between samples prepared with different solvents was observed. As the main motions in the sample at this temperature are attributed to the motions of the hexyl side chains, this result suggests that crystallinity of the sample has very little affect on the side chain dynamics. Additionally it was found that when comparing the dynamics for pure P3HT and the P3HT/PCBM blends, the elastic intensity and mean square displacement for the blends were higher and lower respectively, strongly suggesting that inclusion of PCBM hinders the movement of the side chains. This result was evident at $333 \mathrm{~K}$ and even more strongly apparent at $433 \mathrm{~K}$, as can be seen in Figure 5 where the elastic peak $(\Delta E=0)$ has been broadened for pure P3HT compared to the blends.

In 2016 the same researchers continued their work by using another key feature of neutrons, that they can be polarised, to separate the coherent/incoherent scattering contributions from their samples. 17 The QENS signal relies on incoherent scattering, with coherent scattering leading to increased background levels and reduced signal intensity. For a 1:1 blend the incoherent and coherent contributions to scattering are $75.5 \%$ and $5.5 \%$ for P3HT respectively, and $14 \%$ and $5 \%$ for PCBM respectively. This more recent study was designed to examine whether any part of the reduced elastic intensity previously reported for P3HT/PCBM blends was caused simply by the increased coherent scattering fraction from PCBM. The data confirmed that blending P3HT with PCBM constrained motions of the polymer, with neither the pure sample or the blend showing any coherent dynamics. Neutron polarisation analysis such as this can be vital for cases where the coherent scattering cross-sections are not negligible when compared to the incoherent scattering crosssections. However polarisation adds complexity to the measurement and the subsequent data analysis as well as having a strongly detrimental affect on neutron flux, leading to greatly increased measurement and analysis costs.

Following on from this work, Etampawala, et al. published a report on molecular relaxation and dynamics in $\mathrm{P} 3 \mathrm{HT} / \mathrm{PCBM}$ blends as a function of PCBM loading. [18 The samples specifically comprised PCBM loadings that were low enough to ensure a single phase sample with the PCBM homogeneously distributed throughout the P3HT, i.e. below 20 vol.\% PCBM. Firstly it was found that the pure components each demonstrated a single relaxation process within the limits of the experiment, whereas the blends exhibited two relaxation processes; one fast process attributed to P3HT dynamics, and one slower process attributed to PCBM dynamics. It was also determined that there was a threshold loading of PCBM in P3HT (between 5 and 9 vol.\% PCBM) below which steric 


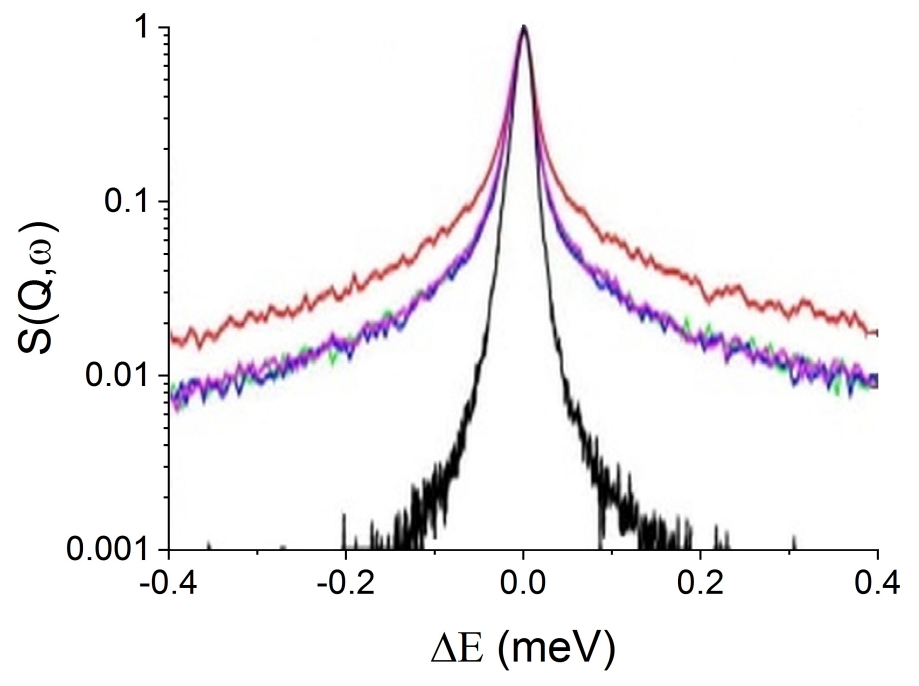

Figure 5: Measured dynamical structure factor as a function of energy transfer for pure P3HT polymer (red line) and 50/50 blends of P3HT/PCBM (at fixed $\mathrm{Q}=1.01 \AA^{-1}$ and $\mathrm{T}=160 \mathrm{~K}$ ). The blends were cast with different solvents; chloroform (blue line), chlorobenzene (green line), and ortho-dichlorobenzene (pink line). The black curve represents the resolution of the instrument (black line). Note the log-scale of the vertical axis and each curve is normalised so that the peak elastic intensity $=1$. Thus a lessening of the elastic peak intensity is apparent as a broadening of the peak and an increase in the intensity of the background signal. The data here show that by blending P3HT with PCBM the dynamics of the system are reduced compared to that of P3HT alone. Adapted with permission from ref. 15 .

hindrance caused by the PCBM dominates the P3HT side chain dynamics. Above this threshold concentration thermal effects dominate the hexyl group motions.

The study of P3HT/PCBM blends was also undertaken by Guilbert, et al. and reported in two related publications. 19, 20 The group used selective deuteration of the P3HT side chains in order to isolate and identify the different motions within their samples. The results were in agreement with those of Paternó, et al. already discussed, above. In addition to the frustrated P3HT motions, Guilbert and co-workers discovered that the polymer also imparted a plasticising effect on the PCBM portion of the blend, which was especially apparent above the glass transition temperature of the polymer, 19 across all experimentally accessible timescales (5-50 ps). The QENS experiment was then used to validate their molecular dynamics simulations. This allowed the group to more confidently extrapolate their theoretical model to time scales 3 orders of magnitude longer then the measured data (up to $50 \mathrm{~ns}$ ), elucidating information would not otherwise be accessible experimentally. 20 This work built upon work previously published by the same group in 2015, in which the researchers demonstrated that QENS could be used to validate molecular dynamics calculations of P3HT and poly(3-octylthiophene) (P3OT, Figure 11. 21] Additionally, this earlier study allowed the determination of activation energies for various reorientations of the polymer side chains. It was concluded that the small differences in the dynamics between P3HT and P3OT are unlikely to account for the superior efficiency of photovoltaic devices made with the former material, thus emphasising the need for further study.

Thiophene-based polymers were also the topic of a study by Díaz-Paniagua, et al. who investigated the molecular dynamics of mixtures of P3OT and functionalised single wall carbon nanotubes in toluene- $d 8$ solutions. 22 The aim of the study was to better understand the sol-gel behaviour and colloid dynamics of blend solutions, which are frequently used in the fabrication of organic electronic devices. The researchers found that below a critical concentration the colloid dynamics are mainly driven by the solvent, and above this critical concentration they are dominated by the solute 


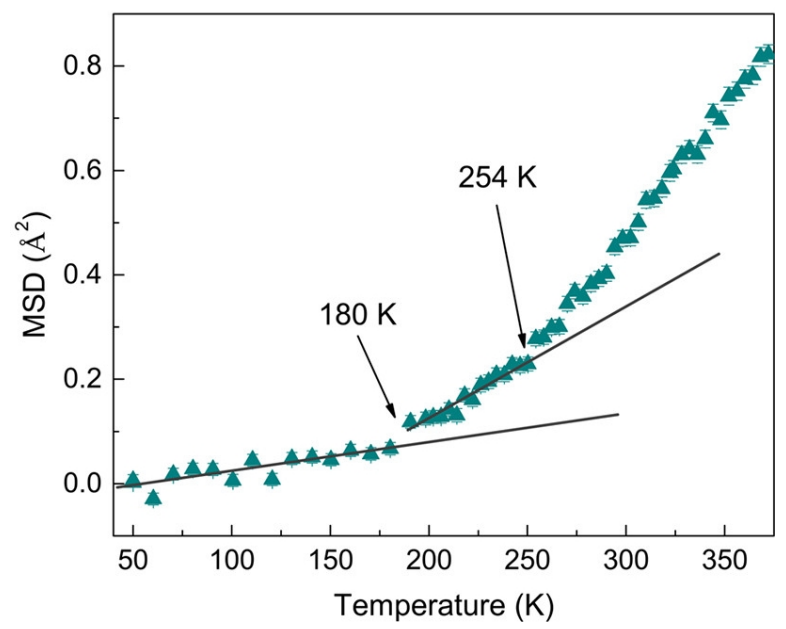

Figure 6: Mean-square displacement (MSD) as a function of temperature for the F4MCTCNQ doped P3HT- $d$ blend, showing the onset of two different dynamical processes at $180 \mathrm{~K}$ and $254 \mathrm{~K}$. Reproduced with permission from ref. 23 .

(polymer). Crucially, this critical concentration for P3OT was found to be within the range often used during device fabrication, $2-3 \mathrm{wt} \%$.

$\mathrm{Li}$, et al. took a slightly different approach to using QENS with organic electronics by examining the diffusion of small-molecule acceptor dopants in organic semiconductor films. 23] The group used a combination of laser scanning confocal fluorescence microscopy and QENS to investigate both the macroscopic and microscopic migration of the dopant molecule tetrafluoromethyloxycarbonyltricyanoquinodimethane (F4MCTCNQ, Figure 1) within films of P3HT. This diffusion is particularly important when using doping-induced solubility control during patterning and device fabrication. F4MCTCNQ was used in place of F4TCNQ in order to increase the number of ${ }^{1} \mathrm{H}$-atoms in the molecule being investigated. The group also used deuterated P3HT (P3HT- $d$ ) so that the motions of the dopant molecule would be the dominant signal during the QENS experiments. By determining the proton mean-square displacement (MSD) as a function of sample temperature (Figure 6) a second change in gradient above the glass transition temperature of P3HT (around $180 \mathrm{~K}$ ) suggested that a previously unknown dynamical process was occurring in the blends at temperatures above $254 \mathrm{~K}$. By using a jump diffusion model to further analyse the QENS data it was discovered that this process likely involved two dynamic components, one fast

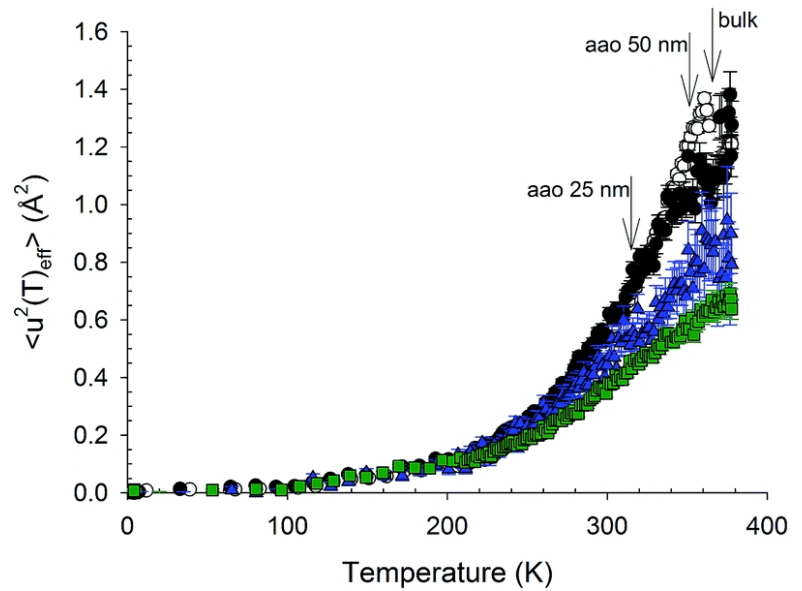

Figure 7: Mean square displacement measured on $\mathrm{Py}(\mathrm{CEH})_{4}$ in the bulk (open circles) or confined in alumina membranes of 50 (solid circles) and $25 \mathrm{~nm}$ (solid triangles) pore diameters, and porous silicon (solid squares). The clearing points of the different samples, determined independently, are marked by the arrows. Reproduced with permission from ref. 24.

component and one slow component. Yet deeper analysis of the data and comparison with DFT calculations suggested that these likely corresponded to methyl group rotations and methoxycarbonyl group rotations respectively, but not translational diffusion as might have been expected.

It is not only polymers and polymer blends that have been examined by QENS. In 2014 Ndao, et al. investigated the molecular dynamics of pyrene based discotic liquid crystals, pyrene-1,3,6,8-tetrarac-2-ethylhexyl tetracarboxylate $\left(\mathrm{Py}(\mathrm{CEH})_{4}\right.$, Figure 1). 24] By impregnating these columnar discotic liquids into solid nanoporous templates the molecules form nanowires, exhibiting translational order along the column axis accompanied by strong overlap of the aromatic $\pi$ orbitals. This orbital overlap leads to long-range transport of charge-carriers in one dimension. Understanding the dynamics of these systems would help with their design and provide new opportunities to tailor charge transport properties. It was found that in the crystal phases, below $220 \mathrm{~K}$, mean square displacements for both confined and unconfined samples were essentially the same and reflected dynamics that are dominated by the rotations of methyl groups at the side chain ends. Above this temperature, in the columnar phases, mean square displacement scaled with pore size, and thus, greater confinement led to reduced motion, as may 
be expected. Interestingly, the clearing temperature for the samples varied dramatically with the level of confinement, with the more strongly confined samples entering an isotropic phase at lower temperatures than the bulk unconfined material (see Figure 7). This is contrary to what may naturally be expected as the samples confined in smaller pores were, at the same temperature, in a more locally disordered state than those in larger pores (or unconfined). The group postulated that the small pore samples experienced highly distributed (and possibly lowered) activation energy barriers, thus increasing local molecular disorder despite the amplitude of the overall dynamics remaining lower than the large pore and unconfined samples, leading to the unexpected observed behaviour.

It is clear to see that much (but not all) of the work discussed in this section has focused on the archetypical P3HT:PCBM photovoltaics system. However, QENS has not yet seen much use in organic transistor or organic LED research to date, highlighting vast areas of potential applications.

\section{Summary and Outlook}

The objective of this article is to highlight some neutron spectroscopy techniques that have not yet been wholly adopted by many researchers studying organic electronics. These techniques provide unique information, such as low-energy vibrational modes, the nature of molecular relaxation mechanisms, and the molecular dynamics of organic systems that would be difficult or impossible to obtain using other methods. The work that has been discussed demonstrates some of the methods by which the key properties of neutrons and neutron spectroscopy can be used to uniquely address the questions facing researchers of organic electronics.

Neutron spectroscopy is not without its weaknesses. Sample sizes are often larger than those needed for other techniques, 0.1-10 $\mathrm{g}$ is typical. Additionally due to the weak interaction of neutrons with most matter the data acquisition times are often on the order of minutes-hours rather than seconds (or less) typical for photon-based measurements. However, neutron sources and instrument designs are constantly improving leading to reduced measurement times, reduced sample sizes, and finer resolution spectra.

INS and QENS have a strong relationship with theoretical calculations as discussed above. With the ever-decreasing cost of computational power leading to the ability to perform more accurate calculations involving larger conjugated molecules and polymers, combined with the ever-increasing demand for inexpensive, portable, efficient electronics, it is hoped that these neutron scattering techniques will see even more interest in the future.

Finally, even though neutron spectroscopy is not available in the average research laboratory there are several large-scale facilities situated around the globe. 25 Of particular current interest is the European Spallation Source (ESS), currently under construction in Lund, Sweden, and due to begin initial operations and instrument commissioning within the next 3-4 years. 26 Access to the vast majority of facilities that offer neutron scattering is freely available to researchers, usually on the proviso the results will be published in the open literature. This means the techniques discussed here are free at the point of use and can be used to elucidate the most important scientific information, regardless of means.

\section{Acknowledgements}

The author would like to thank Stewart F Parker and Victoria García-Sakai for their productive discussions during the writing of this article.

\section{Keywords}

Neutron, Spectroscopy, Organic Electronics, Inelastic Neutron Scattering, Quasielastic Neutron Scattering

\section{References}

[1] R. Pynn, Neutron Scattering A Primer. Los Alamos Neutron Science Center, 1990. [Online]. Available: https://www.ncnr.nist.gov/summerschool/ ss17/pdf/NeutronScatteringPrimer.pdf

[2] B. T. M. Willis and C. J. Carlile, Experimental Neutron Scattering. Oxford, New York: Oxford University Press, 2013.

[3] S. F. Parker, D. Lennon, and P. W. Albers, "Vibrational Spectroscopy with Neutrons: A 
Review of New Directions," Appl Spectrosc, vol. 65, no. 12, pp. 1325-1341, 2011.

[4] J. P. Embs, F. Juranyi, and R. Hempelmann, "Introduction to Quasielastic Neutron Scattering," Zeitschrift für Physikalische Chemie, vol. 224, no. 1-2, pp. 5-32, 2010.

[5] V. F. Sears, "Neutron scattering lengths and cross sections," Neutron News, vol. 3, no. 3, pp. 26-37, 1992.

[6] P. C. H. Mitchell, S. F. Parker, A. J. RamirezCuesta, and J. Tomkinson, Vibrational Spectroscopy with Neutrons, ser. Neutron Techniques and Applications. World Scientific Publishing Co Pte Ltd, 2005, no. 3.

[7] P. Hermet, J.-L. Bantignies, A. Rahmani, J.L. Sauvajol, and M. R. Johnson, "Density-ofstates of crystalline 2,2'-bithiophene: ab initio analysis and comparison with inelastic neutron scattering response," J. Phys.: Condens. Matter, vol. 16, no. 41, p. 7385, 2004.

[8] P. Hermet, S. Lois-Sierra, J.-L. Bantignies, S. Rols, J.-L. Sauvajol, F. Serein-Spirau, J.P. Lere-Porte, and J. J. E. Moreau, "Lattice Dynamics of Oligo(phenylenethienylene)s: A Far-infrared and Inelastic Neutron Scattering Study," J. Phys. Chem. B, vol. 113, no. 13, pp. 4197-4202, 2009.

[9] O. Kruglova, F. M. Mulder, A. Kotlewski, S. J. Picken, S. Parker, M. R. Johnson, and G. J. Kearley, "A compact model system for electron-phonon calculations in discotic materials," Chem. Phys., vol. 330, no. 3, pp. 360-364, 2006.

[10] A. M. Pivovar, J. E. Curtis, J. B. Leao, R. J. Chesterfield, and C. D. Frisbie, "Structural and vibrational characterization of the organic semiconductor tetracene as a function of pressure and temperature," Chem. Phys., vol. 325, no. 1, pp. 138-151, 2006.

[11] T. F. Harrelson, Y. Q. Cheng, J. Li, I. E. Jacobs, A. J. Ramirez-Cuesta, R. Faller, and A. J. Moule, "Identifying Atomic Scale Structure in Undoped/Doped Semicrystalline P3ht Using Inelastic Neutron Scattering," Macromolecules, vol. 50, no. 6, pp. 2424-2435, 2017.
[12] T. F. Harrelson, V. Dantanarayana, X. Xie, C. Koshnick, D. Nai, R. Fair, S. A. Nuez, A. K. Thomas, T. L. Murrey, M. A. Hickner, J. K. Grey, J. E. Anthony, E. D. Gomez, A. Troisi, R. Faller, and A. J. Moul, "Direct probe of the nuclear modes limiting charge mobility in molecular semiconductors," Mater. Horiz., 2018.

[13] V. García-Sakai and A. Arbe, "Quasielastic neutron scattering in soft matter," Current Opinion in Colloid 8 Interface Science, vol. 14, pp. 381-390, 2009.

[14] J. Obrzut and K. A. Page, "Electrical conductivity and relaxation in poly(3hexylthiophene)," Phys. Rev. B, vol. 80, no. 19, p. 195211, 2009.

[15] G. Paterno, F. Cacialli, and V. García-Sakai, "Structural and dynamical characterization of P3HT/PCBM blends," Chem. Phys., vol. 427, pp. 142-146, 2013.

[16] Y. Zhao, G. Yuan, P. Roche, and M. Leclerc, "A calorimetric study of the phase transitions in poly(3-hexylthiophene)," Polymer, vol. 36, no. 11, pp. 2211-2214, 1995.

[17] G. M. Paterno, J. R. Stewart, A. Wildes, F. Cacialli, and V. García-Sakai, "Neutron polarisation analysis of Polymer: Fullerene blends for organic photovoltaics," Polymer, vol. 105, pp. 407-413, 2016.

[18] T. Etampawala, D. Ratnaweera, B. Morgan, S. Diallo, E. Mamontov, and M. Dadmun, "Monitoring the dynamics of miscible P3HT:PCBM blends: A quasi elastic neutron scattering study of organic photovoltaic active layers," Polymer, vol. 61, pp. 155-162, 2015.

[19] A. A. Y. Guilbert, M. Zbiri, M. V. C. Jenart, C. B. Nielsen, and J. Nelson, "New Insights into the Molecular Dynamics of P3HT:PCBM Bulk Heterojunction: A Time-of-Flight QuasiElastic Neutron Scattering Study," J. Phys. Chem. Lett., vol. 7, no. 12, pp. 2252-2257, 2016.

[20] A. A. Y. Guilbert, M. Zbiri, A. D. F. Dunbar, and J. Nelson, "Quantitative Analysis of the Molecular Dynamics of P3HT:PCBM Bulk 
Heterojunction," J. Phys. Chem. B, vol. 121, no. 38, pp. 9073-9080, 2017.

[21] A. A. Y. Guilbert, A. Urbina, J. Abad, C. DíazPaniagua, F. Batallán, T. Seydel, M. Zbiri, V. García-Sakai, and J. Nelson, "TemperatureDependent Dynamics of Polyalkylthiophene Conjugated Polymers: A Combined Neutron Scattering and Simulation Study," Chem. Mater., vol. 27, no. 22, pp. 7652-7661, 2015.

[22] C. Díaz-Paniagua, A. Urbina, V. GarcíaSakai, T. Seydel, J. Abad, J. Padilla, R. García-Valverde, N. Espinosa, M.-J. GómezEscalonilla, F. Langa, and F. Batallán, "Molecular dynamics of solutions of poly-3-octylthiophene and functionalized single wall carbon nanotubes studied by neutron scattering," Chemical Physics, vol. 427, pp. 129-141, 2013.

[23] J. Li, C. Koshnick, S. O. Diallo, S. Ackling, D. M. Huang, I. E. Jacobs, T. F. Harrelson, K. Hong, G. Zhang, J. Beckett, M. Mascal, and A. J. Moule, "Quantitative Measurements of the Temperature-Dependent Microscopic and Macroscopic Dynamics of a Molecular Dopant in a Conjugated Polymer," Macromolecules, vol. 50, no. 14, pp. 5476-5489, 2017.

[24] M. Ndao, R. Lefort, C. V. Cerclier, R. Busselez, D. Morineau, B. Frick, J. Ollivier, A. V. Kityk, and P. Huber, "Molecular dynamics of pyrene based discotic liquid crystals confined in nanopores probed by incoherent quasielastic neutron scattering," $R S C A d v$., vol. 4, no. 103, pp. $59358-59369,2014$.

[25] "Neutronsources.org." [Online]. Available: https://neutronsources.org/

[26] R. Garoby, A. Vergara, H. Danared, I. Alonso, E. Bargallo, B. Cheymol, C. Darve, M. Eshraqi, H. Hassanzadegan, A Jansson, I. Kittelmann, Y. Levinsen, M. Lindroos, C. Martins, . Midttun, R. Miyamoto, S. Molloy, D. Phan, A. Ponton, E Sargsyan, T. Shea, A. Sunesson, L. Tchelidze, C. Thomas, M. Jensen, W. Hees, P. Arnold, M. JuniFerreira, F. Jensen, A Lundmark, D. McGinnis, N. Gazis, J. W. II, M. Anthony, E. Pitcher, L. Coney, M. Gohran, J. Haines, R. Linander, D Lyngh, U. Oden, H. Carling, R. Andersson,
S. Birch, J. Cereijo, T. Friedrich, T. Korhonen, E. Laface, M Mansouri-Sharifabad, A. MoneraMartinez, A. Nordt, D. Paulic, D. Piso, S. Regnell, M. Zaera-Sanz, M. Aberg, K Breimer, K. Batkov, Y. Lee, L. Zanini, M. Kickulies, Y. Bessler, J. Ringnr, J. Jurns, A. Sadeghzadeh, P. Nilsson, M Olsson, J.-E. Presteng, H. Carlsson, A. Polato, J. Harborn, K. Sjgreen, G. Muhrer, and F. Sordo, "The European Spallation Source Design," Phys. Scr., vol. 93, p. 014001, 2018. 